\title{
SOME POINTS OF DIFFERENCE CONCERNING THE THEORY OF MUSIC.
}

\author{
BY PROFESSOR MAX MEYER,
}

\section{University of Missouri.}

Mr. Dixon's excellent criticism in Mind ${ }^{1}$ of my theory of music $^{2}$ suggested to me to write the following pages, which, I hope, will make some details of my theory clearer than I was able to make them in the publication which he reviewed. I shall further have to refer to an article of Lipps ${ }^{3}$ and an earlier one of my own ${ }^{4}$ and therefore mention them here at once.

Mr. Dixon states that according to my experience melodic relationship does not exist between two tones the ratio of whose vibrations involves primes higher than 7 . The latter part of this statement does not quite agree with what I wished to say. One must not infer that there is relationship whenever the primes involved are lower than II; I do not believe to experience, e. $g$., relationship with $7-9$, or $7-15$. I am extremely glad, however, for his use of the word experience. My theory, indeed, is not intended to be a mere dialectic, but the systematized expression of my experience. I regret as much as the reviewer that I have not tried my experiments on a large number of unbiased observers. This is not caused by any belief in my own self-sufficiency, but by circumstances over which I have no control. To find unbiased observers for experiments of this kind is much more difficult than it seems to be. Further, the methodical difficulties of such experiments are extraordinarily great; and to make the experiments in such a way as they ought to be made requires an amount of time of which those

'Mind, New Series, 44, Oct., I902, pp. 567-571.

2Max Meyer, 'Contributions to a Psychological Theory of Music,' University of Missouri Studies, I., I, IgOI, pp. I-80.

'Th. Lipps, 'Zur Theorie der Melodie,' Zeitschrift für Pychologie und Physiologie der Sinnesorgane, Bd. 27, 190r, pp. 225-263.

'Max Meyer, 'Elements of a Psychological Theory of Melody,' PsychoLOGICAI, REVIEW, VII. (3), 1900, pp. 24 I-273. 
have no conception who never attempted it. The chief cause why I did not furnish my theory with a greater material of data is the well-known fact that a college professor has plenty of other duties besides making experiments. Therefore the experience referred to is mostly my own experience; but this does not mean that it is only a casual observation. I have become convinced of the value of my theory, and I regard this a sufficient reason for publishing it. Nevertheless I may be wrong in many respects, and $I$ shall be thankful for any objections brought forward in so truly a scientific spirit as the reviewer's.

The melodic relationships which I believe to experience are expressed by the symbols: 2-2, 2-3, 2-5, 3-5, 3-7, 2-7, 2-9, $2-15,5-9$ and 5-7. No account is taken of 2 as a factor. This is of the utmost importance, as it simplifies the theory immensely. It is interesting to me to see that a mathematically trained mind like Mr. Dixon's does not object to this simplification, whereas another reviewer called this omission of all factors which are pure powers of 2 unwarranted, without being aware, obviously, of the fact that musicians for centuries have done what amounts practically to the same in naming all tones by the same letter which differ by multiples of an octave. This fact, with which he was familiar since his earliest youth, seemed to him quite natural; but my omission of the powers of 2 he could not grasp and therefore rejected it.

It seems to me that I have been greatly misunderstood with respect to my psychological definition of a 'tonic.' This tonic is not identical with what musicians call a 'key-note.' The word key-note, as I would like to use it, refers only to the system of musical notation which we desire to employ in order to write down a certain tune; to the sharps and flats which we use. It has no psychological significance - i. e., in my writing. I expected to make this clear by not usually employing the common musical notation. If we use the common musical notation, if we distinguish a tone by calling it key-note, we should of course select as a key-note a tone which is psychologically emphasized in the melody above the others. But since this emphasis may be based on very different psychological facts I shall not here introduce 'key-note' as a scientific term. A scientific 
term must have a distinct meaning, and not more than one meaning. My definition of a 'tonic' is correctly quoted by Mr. Dixon: It is a pure power of 2 when combined with melodically related tones, $i$. $e_{\text {., }}$ with $3,5,7,9$ or 15 . Such a combination of successive tones we wish to have end on no other tone but the power of 2 . I do not mean that, physically, one cannot close on one of the other tones; of course one may, but he produces then an æsthetic effect that is hardly ever desired. Further, I do not mean that, whenever we are satisfied by a certain tune ending on a certain note, this note must be a tonic as above defined. It may be a tonic only relative to some of the tones contained in the whole melody; not relative to others, not to the melody as a whole. Indeed, it may not be a tonic even relative to a single other tone, and yet we may be satisfied by the ending, perhaps for no other reason but that we know that this tune ends on this tone, and if it did not, it would not be the tune we expect to hear. This multiplicity of the causes of a certain æsthetic experience is the greatest of all the obstacles to experimental research in this field. This will do away with Mr. Dixon's objection to my theory that it necessitates the use in a complex piece of music of very large numbers to represent the tones. "If, for example, a melody, in ordinary language, modulates to the subdominant key, Professor Meyer would say: $2 I$ becomes a partial tonic. The subdominant of the new key would then be represented by $44 \mathrm{I}$, which contains the square of 7, and it is not given in Professor Meyer's scale." Herein I cannot agree with the reviewer. I omitted the square of $\eta$ in the complete scale because, after a good deal of experimental work, I became convinced, and am convinced, that the square of 7 is never used in actual music. I should not say that 2I becomes a partial tonic, since I should not represent this subdominant by 21 , nor the key-note in this case by 2 . On the contrary, I should say that this piece of music, as a whole, has no tonic. What this means I hope to make clearer by the following.

I define the complete musical scale as the infinite series of all products of the powers of $2,3,5$ and 7 ; because within this scale is to be found any melody imaginable. Of course, we cannot write down a complete infinite series. Let us here write 
down the series up to 405. The reader will see that for the present purpose this is sufficient. Let us also omit all those numbers which are derived from smaller ones by multiplication with a power of 2 . We do not need them here. We then have the following series:

$2,3,5,7,9,15,21,25,27,35,45,49,63,75,8 \mathrm{I}, 105$, $125,135,175,189,225,243,315,375,405$.

It seems to be a simple fact that the æsthetic effect of a melody depends on both these conditions : variety and closeness of relationship. (I do not mention here the tonic effect, since this may be absent.) But Lipps, Dixon and others add a further condition for a succession of tones to be a melody. $\mathrm{Mr}$. Dixon says : "To apprehend a succession of tones as a melody is, psychologically, to apprehend relationships which imply the relation of each note to a tonic, just as on the physical side to represent the notes by numbers having simple numerical ratios is to imply a number which is the greatest common measure of all." I must say, on empirical grounds, that this seems to me an $a$ priori assumption. I do not find it necessary that in a melody all tones must be related to a certain single tone, but merely that each tone must be related to some other tone, and any group of tones to some other group, temporally near enough, so that the relationship can take effect. Neither can I admit that the greatest common measure has anything to do with musical theory; else, why should there be relationship between the tones 15 and 16 and no relationship between the tones Io and II? To speak of the greatest common measure means to go beyond the facts actually observed. This is one of the reasons why I used as symbol for the power of 2 the figure 2 and not I; I anticipated that the figure I would falsely suggest something like the greatest common measure.

The conditions of melodic effect, variety and closeness of relationship are to some extent opposed to each other. If we use for a melody six different tones in intervals of octaves, there is the greatest closeness of relationship, but no variety; there is only a single relationship in the melody, namely, 2-2. 
If we play the tones successively as we find them in the complete scale, there is enough variety of relationship, but hardly any closeness to speak of. Actual music, therefore, will be a compromise of these two conditions. This consideration can help us to derive from the complete scale smaller scales, which are more easily applicable to special pieces of music.

Let us determine the greatest number of tones which are all mutually related. Here, however, we may either make use or not of the particular effect of a power of 2 when combined with a related tone, the tonic effect, as $I$ have called it. Let us first use this effect in the strongest possible manner, and then try the other way of avoiding this effect as far as this is possible.

Which, then, is the greatest number of tones, including 2, which are all mutually related? I must suppose, of course, that the reader will always keep in mind those relationships which I experimentally determined: $2-2,2-3,2-5,3-5 ; 2-7$, $3-7,2-9 ; 2-15,5-7,5-9$. Now, if we look over the complete scale as we find it above, we have to accept 2,3 and 5 . But we cannot accept all of the next three tones, 7,9 and 15 , because 7 is not related to either 9 or 15 . We therefore have to choose between 7 on the one side, 9 and ${ }^{5} 5$ on the other. Since the addition of two tones makes the number of tones greater than the addition of one, the above condition compels us to accept 9 and 15 and to reject 7 . We then have the five tones $2,3,5,9$, I5, which are all mutually related. No further tone of the complete scale is related to all of these five.

We agreed to make the tonic effect as strong in these scales as possible. The best method of introducing further tones is then, obviously, to use as a secondary tonic the tone which is most closely related to the tonic. Of the four tones $3,5,9$ and I5 the tone 3 has by far the closest relationship to 2 . If we make 3 a secondary tonic and use relationships of the first degree only, 9 and 15 offer themselves. But they are already in the scale and need not be added. If we use relationships of the second degree we have to add 21 and 27 . Our scale is then the following :

$$
2,3,5,9,15,21,27 \text {. }
$$


If we use also relationships of the third degree, we have to add 45 . Our scale is then this :

$2,3,5,9,15,21,27,45$.

He who desires may add further tones by making another of the original tones a secondary tonic. If he does so, he will become able to have within the whole melody a greater number and a greater variety of partial melodies, each containing a secondary tonic. According to my experience, however, music with a primary tonic is hardly ever very complicated, so that the addition of further tones to the scale is not of much practical significance. The table below permits us to compare the different scales. That 2 has been given the musical name $F$ is quite arbitrary.

Scalas with a Prasary Tonic.

$\begin{array}{lllllllllllll}\text { F } & \text { F\# } & \text { G } & \text { G\# } & \text { A } & \text { A } & \text { B } & \text { C } & \text { C\# } & \text { D } & \text { D\# } & \text { E } & \text { F } \\ 2 & - & 9 & - & 5 & - & - & 3 & - & - & - & 15 & 2 \\ 2 & - & 9 & - & 5 & 21 & - & 3 & - & 27 & - & 15 & 2 \\ 2 & - & 9 & - & 5 & 21 & 45 & 3 & - & 27 & - & 15 & 2 \\ \text { and so on. }\end{array}$

Our second intention was to determine the greatest number of tones mutually related, while neglecting as much as possible the peculiar effect of a tonic. We therefore omit 2 entirely and accept three and five. 7 has to be omitted again, because its acceptance would necessitate the omission of the two tones 9 and 15 , with which 7 is not related. We have further to add 45 , this being related to $3,5,9$ and 15 . No further tone of the complete scale is related to all of these five :

$3,5,9,15,45$.

If we desire to introduce further tones and yet have the closest relationship possible, we must find out to how many of the $e_{L}^{*}$ above five tones each further tone is related and then select the one or those which show the greatest number of relationships. Below we see the tones of the complete scale which are left, each one marked with as many stars as it shows relationships. $E . g ., 7$ is related to 3 and 5 , but not to $9,15,45$; therefore it has received two stars. And so on.

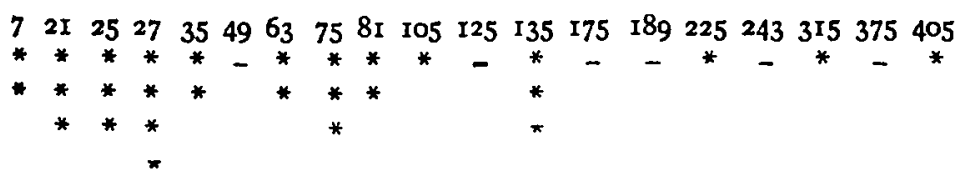


Only 27 has four stars; all the rest have three or less. We therefore add 27 to our scale, which now is the following:

$3,5,9,15,27,45$.

The remaining tones of the complete scale are again marked with as many stars as they show relationships with the tones of this last scale.

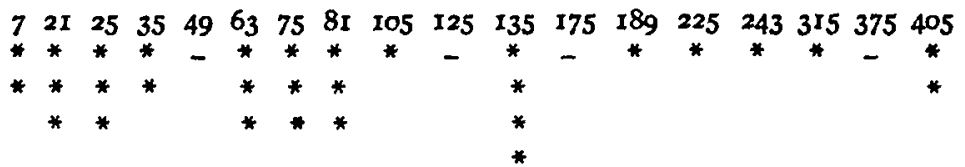

I35 has in this case four relationships; no other tone an equal number. We therefore add $\times 35$ and have the scale :

$3,5,9,15,27,45,135$.

The remaining tones are the following :

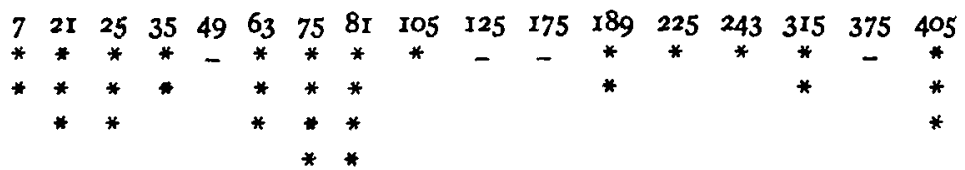

75 and 81 have in this case more relationships than the rest. We therefore add 75 and $8 \mathrm{I}$ :

$3,5,9,15,27,45,75,81, x_{35}$.

The remaining tones are :

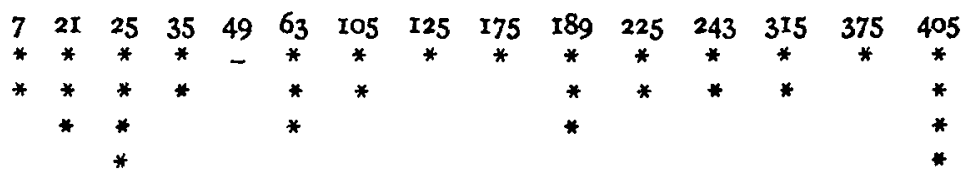

25 and 405 have more relationships than the rest and are added to the above scale :

$3,5,9,15,25,27,45,75,8 \mathrm{r}, \mathrm{r} 35,405$.

The remaining tones are :

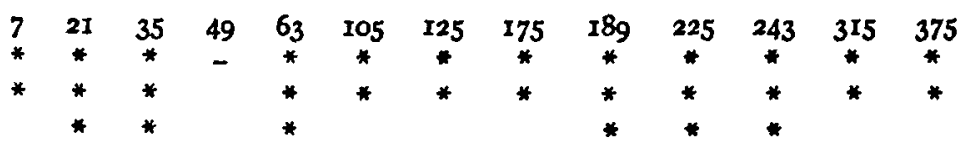

$21,35,63,189,225$ and 243 have three relationships each and are added to the last scale: 
$3,5,9,15,21,25,27,35,45,63,75,81,135,189,225$, 243,405 .

If one wants to continue this, he must take into consideration a greater part of the complete scale than we did. We stopped at 405. But a greater extension of these scales is of little practical significance. The table below permits us to compare these scales.

\begin{tabular}{|c|c|c|c|c|c|c|c|c|c|c|c|c|}
\hline & & & SCAI & ES & $\mathrm{TrH}$ & $\mathrm{IT} A \mathrm{~F}$ & RIM & $R Y T$ & NIC. & & & \\
\hline G & G\# & A & $A \#$ & B & C & $C \#$ & $D$ & $\mathbf{D} \#$ & $\mathbf{E}$ & $F$ & $\mathbf{F} \#$ & G \\
\hline 9 & $\rightarrow$ & 5 & - & 45 & 3 & - & - & - & I5 & - & - & 9 \\
\hline 9 & - & 5 & - & 45 & 3 & - & 27 & - & I5 & - & - & \\
\hline 9 & - & 5 & - & 45 & 3 & - & 27 & - & I5 & - & I35 & \\
\hline 9 & 75 & $\left(\begin{array}{r}5 \\
8 \mathrm{r}\end{array}\right)$ & - & 45 & 3 & 一 & 27 & - & 15 & - & 135 & \\
\hline 9 & 75 & $\left(\begin{array}{r}5 \\
8 \mathrm{r}\end{array}\right)$ & - & 45 & 3 & $\left(\begin{array}{r}25 \\
405\end{array}\right)$ & 27 & - & 15 & - & 135 & \\
\hline 9 & 75 & $\left(\begin{array}{r}5 \\
81\end{array}\right)$ & $2 I$ & 45 & & $\left(\begin{array}{r}25 \\
405\end{array}\right)$ & 27 & $225^{\circ}$ & $\left(\begin{array}{r}15 \\
243\end{array}\right)$ & 63 & I35 & \\
\hline
\end{tabular}

and 80 on.

An important fact, to be learned theoretically from the above scales, is the relative insignificance of 7 , although the complete omission of 7 would prevent a truly scientific theory of music. In the scales with a primary tonic the pure 7 is absent; so is it in the scales without a primary tonic. In the latter the products containing the factor $7, i$. e., $21,35,63,189$, enter into the scales according to closeness of relationship only comparatively late, after many other tones. The square of 7 never enters into any. of these scales. This agreement of my theory with the generally recognized fact of the comparative insignificance of the number 7 for musical theory proves the correctness of the principles of my theory, the correctness of my observations concerning the laws of relationship of any two tones.

In the scales with a primary tonic there can be no question as to which tone should be the key-note; of course the tonic. But in the scales without a primary tonic this depends or may depend on many different conditions. The tones which are most commonly used as key-notes are 9 and 5. Why these tones are used thus, I shall now try to make clear.

Let us study more closely the relationships contained in the series $3,5,9,15,27,45,75,8 I, 135$. There is no primary tonic, but several of the tones are secondary tonics. We re- 
member that the tonic effect is particularly strong in the relationships $2-3$ and 2-5. Let us therefore see which of the tones of the above series can be combined with tones of these two relationships.

$\begin{array}{rrrrrrrrr}3 & 5 & 9 & 15 & 27 & 45 & 75 & 8 \mathrm{r} & 135 \\ 9 & 15 & 27 & 45 & 8 \mathrm{I} & 135 & & & \\ 15 & & 45 & 75 & 135 & & & & \end{array}$

We see that only $3,9,15$ and 27 can be combined with tones of both the relationships $2-3$ and $2-5$. Let us for the present discussion call these four groups of three tones each by the names group 3, group 9, group I5 and group 27. We should now determine how these groups are interrelated. Each combination of two groups makes nine relationships possible. The table below shows how many of these nine are lacking.

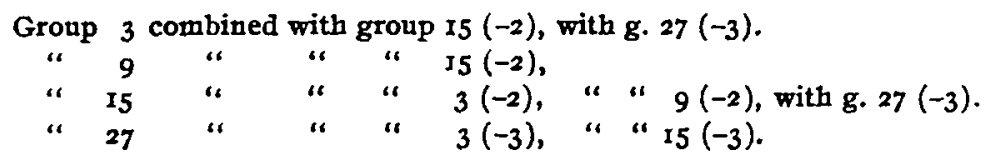

The tones of group 3 and group 9 are all mutually related. Group 3 combined with group 55 lacks two relationships, 75 not being related to either 3 or 9 . This is expressed by the parenthesis $(-2)$. Group 3 combined with group 27 lacks three relationships, 3 not being related to either $8 \mathrm{I}$ or $\mathrm{I}_{35}$, I 5 not to $8 \mathrm{I}$. This is expressed by the parenthesis $(-3)$. Group 9 combined with group 15 lacks two relationships, 75 not being related to either 9 or 27 . Group 15 combined with group 27 lacks three relationships, $8 \mathrm{I}$ not being related to either $x 5$ or 75,27 not to 75. The group that is most deficient in relationships to the other groups is therefore 15 ; the one that lacks only two of twenty-seven relationships is the group 9. If we have to emphasize a certain tone melodically, to make it the key-note, nothing is more natural than that we choose the tonic of this group $9-27-45$ as the key-note. Therefore $I$ have made 9 the key-note.

The above explanation is somewhat similar to that of Lipps. The most important difference is that he needs about fifteen pages in order to explain what I explain on a single page. 
Lipps insists on calling the tones 3 and 27 in the above series 'dominants.' Any scientific term should be definable; but I have not been able to discover any definition of 'dominant' which could consistently be used. On the other hand, I do not see of what advantage it is to use the word dominant. Nothing becomes clearer or simpler by using the word. If 3 and 27 are to be distinguished by special names, I do not see why 15 should go without; I propose the name of duke or prince.

It is decidedly dangerous to call 3 and 27 in the above case dominants. Musicians invariably call $F$ and $G$ dominants in any music written in the key of $\mathrm{C}$. But we should remember that $F$ and $G$ in other scales, e.g., the scale 2, 9, 5, 21, 3, 27, 15,2 , have psychological characteristics quite different from those we found here.

Nearly all music that is highly complex, particularly vocal music for a choir of several voices, polyphonic music like organ fugues, and whole operas, seem to be represented by scales without a primary tonic with 9 as a key-note. The scale can of course be further extended than we used it above; remaining, however, within the complete musical scale. Within the complete scale there are many possibilities of partial melodies containing a partial tonic. The greater number of arias in operas are partial melodies of this kind.

Here may be mentioned a fact which has been much discussed by the theorists. It is possible to use in a piece of music written in the key of C (9) both these chords ; D-G-B (8I-27I35) and D-F-A (5-3-15). This does not cause us the slightest theoretical difficulty. We see in our scales that the tone D on the piano is either 5 or $8 \mathrm{I}$. There is no reason why in our music we should not use now the one, now the other. But those whose adhere to the 'diatonic scale' of Zarlino-RameauHelmholtz do not get off so easily, since their scale contains only $8 \mathrm{r}$, not 5 . In order to explain so simple a fact, a single chord, they have to introduce such a complicated theory as that of 'modulation.' Indeed Helmholtz says, though somewhat reluctantly, that this chord D-F-A in the key of $\mathrm{C}$ is 'eine beginnende Modulation über die Grenzen der C-Durtonart hinaus.' If simplicity is a requirement for a theory to be called 
scientific, no further criticism is necessary. I might easily mention a hundred similar cases, if it were not for the waste of paper and printer's ink.

In music without a primary tonic, besides 9,5 is frequently used as a key-note on which a melody ends. I shall now try to answer the question why this ending on 5 is 'satisfactory.' Dixon says in his review: "There are some respects in which Professor Meyer's paper is disappointing. In particular he throws no light on what to the psychologist is one of the most interesting problems presented by music, namely, the peculiar æsthetic effect of minor melodies." It is disappointing to me to read this, as I actually thought to have thrown some light on this problem. I shall try to do so more successfully now. Let us consider the asthetic effect of a melody made up of the tones 3,5 and 15.3 is a tonic relative to 15.5 also is a tonic relative to 15 . When we hear alternately these three tones, each time when 15 sounds we experience the strong desire to hear now 3 ; but also the desire to hear now 5. Fortunately, it is possible to hear both at the same time in a chord. But when we hear both 3 and 5 , our attention is attracted by two sensations almost equally; indeed, we may say equally. The relationship ${ }_{5}-5$ is closer than the relationship ${ }^{5} 5-3$; but not very much. And while this makes the tone 5 psychologically more active than 3, there is another important factor. The relationship between 5 and 3 is very close. I have repeatedly pointed out in other writings that closeness of relationship tends to spread our attention over different tones, prevents our attention from remaining concentrated upon a single tone. (This is very important in the process of analyzing.) If one of the two tones 5 and 3 were a tonic relative to the other, our desire to return to the tonic would render that tone predominant. But this is not the case. Such an experience is something unusual; our usual experiences end in definite reactions. This wavering between two sensations has a strong emotional effect, which may be fittingly described by the German proverb: Wer die Wahl hat, hat die Qual. Our experience is highly ' unsatisfactory.' And yet it is very satisfactory in another way; just as it is satisfactory to see the sufferings of the hero in a drama. 
If we regard one of the three tones of the melody as the keynote, it is natural to take either 5 or 3 , not 15 . We select 5 , because this has the closer relationship to 15 . We may of course construct a melody of more than the three tones 3,5 , and 15. But there are no other three tones in the complete scale (excluding 2) which are united by so close relationships as 3,5 and $\times 5$. The addition of further tones does not, therefore, alter our conclusions as to the tone which should be called the key-note, namely 5 .

Tonic $\left.\begin{array}{ccccccccccccc}\text { C } & \text { C\# } & \text { D } & \text { D\# } & \text { E } & \text { F } & \text { F\# } & \text { G } & \text { G\# } & \text { A } & \text { A\# } & \text { B } & \text { C } \\ \text { Atonic } & & 9 & & 5 & 21 & 45 & 3 & & 27 & & 15 & 2 \\ 9 & 75 & \left(\begin{array}{c}5 \\ 5\end{array}\right) & & 45 & 3 & & 27 & & 15 & 63 & 135 & 9 \\ 5 & & 45 & 3 & 25 & 27 & & 15 & 63 & & 9 & 75 & 5\end{array}\right\}$ Major

The above table shows how the three types of music which we have to distinguish upon psychological grounds are related to the musical notations of music as music in major and minor keys. The three types are represented by three arbitrarily selected series of tones of which a melody of each type may be constructed. Of course, fewer tones may be used or further tones be added. The table shows that tonic and atonic music are by no means identical with major and minor music. But minor music is never tonic.

In a similar manner as by Dixon, I have been misunderstood by Lipps in his recent article quoted in the beginning of this paper. I regret that Lipps in his discussion of my theory does not sufficiently distinguish between what I mean by 'tonic' and what he means by 'Tonica.' He calls Tonica that tone on which any melody satisfactorily ends. I call tonic that tone which is a pure power of 2 relative to some related tone. Lipps implies that, whenever a melody ends on a certain tone, I call and must call this tone tonic and represent it by a pure power of 2 . This idea has never entered my mind. On the contrary, I believe that the greatest error which can be made in musical theory, is to assume that there is only one cause of a satisfactory ending of a melody. That the final tone is a 'tonic' is one cause for a satisfactory ending; but I have never denied that a melody can satisfactorily end from many other causes. In my first publication concerning this matter (Psychological Review) I 
published the intonation of a melody which ends on 9 . To be sure, the psychological effect of such a melody is different from that of a melody containing and ending on a tonic. But there is not a single word in any of my publications, so far as I am aware, stating that a melody which does not contain a primary tonic (2) and, therefore, does not end on it, for this reason has no satisfactory ending, is disagreeable, is ugly. This is one of the prejudices I am fighting, that a certain æsthetic effect must have a single cause, can have no others. Our psychical processes are not so simple. Lipps then proceeds to assert that according to my theory a succession of tones that does not contain a 'tonic' cannot be a melody at all, since 'the most elementary law of my theory' is that a melody must contain a tonic and since each melody naturally has a final tone, which Lipps calls 'Tonica.' The confusion is here caused by Lipps' unwillingness to grant me the right of calling something tonic which differs from what he calls Tonica. Of course, each melody must have a Tonica, i. e., a final tone. But my 'most elementary law of melodic succession' says nothing of the Tonica, the final tone of any arbitrary melody, but merely states ('Contributions,' p. 24): 'that no hearer is satisfied, if after having heard once or more often the tonic 2 he does not find 2 finally at the end of the melody.' I did not suspect that any one could understand this 'after having heard' otherwise than as a conditional clause.

On page 253 of his paper Lipps proves: Meyer verkennt das Wesen der Melodie. One of the premises of this conclusion is the following: Immer, wenn $\mathbf{C}$ 'Tonica' ist, soll, nach Meyer, $\mathrm{F}$ zu C im Verhältnis von 2I-2, $\mathrm{A}$ zu C im Verhältnis 27-2 stehen. This premise is imaginary. That I do not represent every key-note by 2 , Lipps might have noticed. E. g., in Chapter VI. (4) he might have found that among the numbers representing the whole of Schubert's Heidenröslein 2 does not appear at all, in spite of the fact that the piece has a key-note, a 'Tonica,' being written on page 55 in the key of C. If Lipps should think that my interpretation of his term 'Tonica' is incorrect, I challenge him herewith to give a clear-cut and universally applicable definition of what he means by Tonica. 
A very brief, but equally unconvincing refutation of my theory is to be found on page 234 of Lipps' article in the paragraph ending with the words: Meyer's Theorie ist also falsch. Lipps speaks of a melody made up of the tones $2,3,5$ and 7 . In order to understand the argument, it is well to multiply all the numbers with 3 , so that we have the tones $3,9,15$ and 21 . The relationships are not altered by this arithmetical procedure. Lipps then rightly states that according to my theory a melody made up of the tones $3,9,15,21$ cannot satisfactorily end on any other tone but 3. But, he adds, the melody ends most satisfactorily only on 2. Now, I do not see how, logically, a melody made up of the tones $3,9,15,21$ can end on a tone which is not among those tones of which the melody is made up. To my mind the final tone of a melody is as much a part of that melody as an animal's tail is a part of that animal's body. That a melody made up of the tones $2,3,9,15,21$ can satisfactorily end only on 2, is exactly what $I$ have been preaching all the time, and I am delighted to see this confirmed by Lipps. But I do not see how this proves: Meyer's Theorie ist also falsch. The succession of the tones G-B-d-f-G-c is one which we have heard innumerable times, so that it would be most wonderful if we did not expect, after having heard the separately quite unusual succession G-B-d-f-G, to hear a final c. We should never forget that one effect may have more causes than one.

On page 260 Lipps says, with the apparent intention of contradicting me: Why should not $\mathrm{F}: \mathrm{C}$ in the key of $\mathrm{C}$ be represented by both ratios simultaneously, 21:16 and $2: 3$ ? That my answer to this question would not be unconditionally in the negative, Lipps could know if he would look at the complete scale (this scale was published in order to be used thus), where he can find that, for $\mathrm{C}=9, \mathrm{~F}$ is represented by both $\mathrm{r} 89$ and 3 ; so that in this respect there is perfect agreement between Lipps and me. Only I do not believe that the tone $\mathrm{F}$ on the piano can simultaneously act as 189 and 3 ; this seems to me in contradiction to all our general psychological experience, as I have stated already in my Contributions. But it may act now as 3 , later as 189 , then again as 3 , and so.on. 
Musically trained persons, however, frequently deny that $F$ in the key of $\mathrm{C}$ can ever be $2 \mathrm{I}$ to 2 . Their theory as well as their practice have usually made them so accustomed to the chord $F$ A-C in the key of C, that they cannot help imagining this chord when they hear F. Save this artificially acquired habit, no experiential facts necessitate the use of the chord F-A-C in each and every music written in the key of $\mathrm{C}$. One can write a most beautiful accompaniment of a song made up of the tones 2,3 , $5,9,15,21,27, C=2$, without using at all the chord F-A-C. But musical theorists rarely care for experiential facts. The chord F-A-C is 'the chord of the subdominant,' and not to use it would be irreverence shown to the musical Idol Subdominant. If one insists upon using, in the key of $\mathrm{C}$, the chords C-E-G, G-B-D and also F-A-C, - and there are of course no physical means to prevent any one from doing it - then indeed $\mathrm{C}$ cannot theoretically be 2 or $\mathrm{F} 2 \mathrm{r}$.

In case the chord F-A-C is used, one must select the tones from the series which I have characterized above as atonic and major, the key-note $\mathrm{C}$ being 9. Should we use in the music the tones $9,8 \mathrm{r}, 45,3,27,15, \mathrm{r} 35$, but omit 5 , as we may of course use any smaller number of tones than we find in any of the above series, then my theory seems to be confronted with an apparent difficulty. But the difficulty is merely one of formal logic, not a scientific one. Namely all the symbols can be divided by 3 , and the resulting series is : $3,27,15,2,9,5$, 45. I called this series atonic, but since it contains 2, it seems to be tonic. Logically, this is contradictory; but scientifically, there is no contradiction. The tone 2 , which is numerically a tonic, does not act psychologically as a tonic in this case, because the tonic effect is overpowered by another effect. I have shown on an earlier page that of the three groups C-E-G, G-B-D and F-A-C, or in numerical symbols $9-45-27,27-135-81$ and $3-15-9$, or after division by 3 the groups $3-15-9,9-45-27$ and $2-5-3$, the group C-E-G is by far more closely related to both the other two groups, than G-B-D is to the remaining two, or F-A-C to the remaining two. This closeness of relationship gives to the group C-E-G such a great psychological effect, that the tone $\mathrm{C}$ becomes the chief tone, and the tonic effect of $\mathrm{F}$ 
becomes insignificant. Scientifically, therefore, there is no contradiction. That the tonic effect is actually suppressed is caused by the peculiar combination of relationships in this special case, as above described.

It is a very important fact which the experimenter should never forget, that such habits as the one just mentioned make most musically trained persons perfectly incapable of acting as observers in certain cases of experimental investigation concerning intonation. They are one-sidedly trained and therefore not unbiased.

Lipps says in conclusion: A melody is an oscillation between the key-note, its fourth and its fifth. If he had said, certain melodies are such oscillations, I should consent. But very many are decidedly not such oscillations, but structures of quite different types.

Let me close with some general remarks concerning the method of investigation. There has been, for so many centuries, too much deduction from $a$ priori principles, and too little induction from specialized experience. The deductive, as well as the inductive method, has its advantages and its disadvantages. The inductive method does not lead at once to completeness, as the deductive does, but leaves a great many questions open. So I am blamed by Lipps and others for not answering this or that question. But if the principle of deduction is wrong, the complete theory is a complete failure, whereas the theory resulting from the inductive method is right so far as it goes. I believe that the only way of finding the æsthetic laws of music, and of melody in particular, is to determine experimentally the æsthetically most effective intonation of a melody and then to analyze the melody, in order to see how the different relationships are combined so that this effect results. But when I read publications concerning musical theory, I almost invariably get the impression that the author believes in only one, more or less narrow, principle of explanation and assumes, without having made any experiment at all, that a more effective intonation is simply impossible. I believe that the basis of all experimental work along this line must be the complete musical scale as developed in my publications; not any small scale of arbitrarily 
selected tones. I made a number of experiments - as many as time permitted; I found that some of the melodies contained 2, some did not. As I said in my publication ('Contributions,' p. 20): 'Pitches represented by the number 2 I shall call tonics,' so I expressed my experience by saying: there are some melodies which contain a tonic, some which do not. There is not a single sentence in my publication, in which I identify a 'tonic' with what Lipps calls a 'Tonica,' or what others call a key-note, or what not. If any one assumes this identification, I cannot prevent him from doing so; but I do not wish to be responsible for the consequences. My experimenting taught me that it would be advantageous for my structural analysis of music to distinguish ' 2 ' by a special name, a scientific term; and I believed and still believe that the term 'tonic' is most appropriate. That the word is often used in a different sense, is regrettable; but what word is not used in more than one sense? It certainly is not used in more than one sense in my publication. I openly confess that I have little reverence for a terminology which when seen under the light of experiment discloses no other claim for being respected than the sacredness of old age. 